\title{
A Case of Tyrosine Kinase Inhibitor- Resistant Chronic Myeloid Leukemia, Chronic Phase with ASXL1 Mutation
}

\author{
Osamu Imataki ${ }^{a}$ Tomoya Ishida ${ }^{a}$ b Hiroyuki Kubo ${ }^{a}$ Makiko Uemura ${ }^{a}$ \\ Yasuhito Nanyac Kimihiro Kawakamid Seishi Ogawa ${ }^{c}$ \\ Norimitsu Kadowakia \\ aDivision of Hematology, Faculty of Medicine, Kagawa University, Kagawa, Japan; \\ bKagawa University Hospital Post Graduate Clinical Education Center, Kagawa, Japan; \\ 'Department of Pathology and Tumor Biology, Kyoto University, Kyoto, Japan; \\ ${ }^{\mathrm{d} D e p a r t m e n t}$ of Hematology, Kagawa Prefectural Central Hospital, Kagawa, Japan
}

\author{
Keywords \\ Chronic myeloid leukemia · Tyrosine kinase inhibitor · Drug resistance · Leukemia \\ oncogenesis · Clonal evolution
}

\begin{abstract}
Hematological malignancies, including chronic myeloid leukemia (CML), exhibit ASXL1 mutations; however, the function and molecular mechanism of these mutations remain unclear. ASXL1 was originally identified as tumor suppressor gene, in which loss of function causes myelodysplastic syndrome (MDS). ASXL1 mutations are common and associated with disease progression in myeloid malignancies including MDS, acute myeloid leukemia, and similarly in CML. In MDS, ASXL1 mutations have been associated with poor prognosis; however, the impact of $A S X L 1$ mutations in CML has not been well described. A 31-year-old male was diagnosed as CML-chronic phase (CP). Laboratory findings showed a white blood cell count of $187,200 / \mu \mathrm{L}$, with asymptomatic splenomegaly. Blast count was $5.0 \%$ in peripheral blood and $7.3 \%$ in bone marrow. There was no additional chromosomal abnormality except for $t(9 ; 22)$ (q34;q11.2) by chromosomal analysis. At onset, the Sokal score was 1.4, indicating high risk. The patient received tyrosine kinase inhibitor (TKI) therapy, comprising nilotinib $\sim 600 \mathrm{mg} /$ day, bosutinib $\sim 600 \mathrm{mg} /$ day, ponatinib $\sim 45 \mathrm{mg} /$ day, and dasatinib $\sim 100 \mathrm{mg} /$ day. Nevertheless, after 1.5 years of continuous TKI therapy, the best outcome was a hematological response. Although additional chromosomal aberrations and $A B L 1$ kinase mutations were analyzed repeatedly before and during TKI therapy, known genetic abnormalities were not detected. Thereafter, the patient underwent bone marrow transplantation from an HLA 7/8 matched
\end{abstract}


unrelated donor (HLA-Cw 1 locus mismatch, graft-versus-host direction). The patient achieved neutrophil engraftment, 18 days after transplantation, leading to complete remission with an undetectable level of $B C R-A B L 1$ mRNA. The patient, however, died from graft-versus-host disease and thrombotic microangiopathy after 121 days. Gene sequence analysis of his CML cell before stem cell transplantation revealed ASXL1 mutations. Physiologically, ASXL1 contributes to epigenetic regulation. In the CML-CP patient in this case report, ASXL1 mutation conferred resistance to TKI through obscure resistance mechanisms. Even though a molecular mechanism for TKI resistance in ASXL1 mutation in CML has remained obscure, epigenetic modulation is a plausible mode of $C M L$ disease progression. The clinical impact including prognosis of $A S X L 1$ for $C M L$ is underscored. And the treatment strategy of CML with ASXL1 mutation has not been established. A discussion of this case was expected to facilitate treatment options.

\section{Introduction}

Chronic myeloid leukemia (CML) is a myeloid clonal disease driven by the $b c r-a b l$ fusion gene, which creates a constitutively active tyrosine kinase. This led to the development of $B C R-A B L 1$ tyrosine kinase inhibitors (TKIs), which provided long-term remission and improved life expectancy of TKI-treated CML patients [1]. The mechanism of TKI resistance in $C M L$, caused by mutation of the BCR-ABL1 kinase domain has been extensively investigated [2]. Importantly, the $B C R-A B L 1$ kinase-independent resistance mechanism due to newly acquired mutations or other genetic aberrations has been reported in a minority of TKIresistant CML cases [3]. One such mutation was found in ASXL1, a histone-binding protein located on chromosome 20q11.2, that disrupts chromatin by enhancing or repressing gene transcription [4]. Then $A B L 1$ kinase-dependent resistance may promote a change of a patient's TKI treatment strategy. As a while, however, it is obscure how much impact the BCR-ABL1 kinase-independent resistance brings on CML therapy plan.

Originally identified from sequence analysis of myelodysplastic syndrome (MDS) patients [5], mutations in ASXL1 were a nonspecific genetic abnormality, associated with poor prognosis not only in MDS [6] but also in bcr-abl-negative myeloproliferative neoplasms [6]. Mutations in ASXL1 have been found in the accelerated phase or blast phase [7] and in CML-chronic phase (CP) [8]. Surprisingly, several ASXL1 mutations have also been reported in healthy people [9-11], indicating the pleiotropic nature of ASXL1. We present a case of CML-CP resistant to various TKIs and discuss the association between mutations in ASXL1 and TKI resistance in CML.

\section{Case Presentation}

A 31-year-old male was diagnosed as CML-CP after an annual occupational health check-up revealed leukocytosis (WBC 187,200/ $\mu \mathrm{L}$ ), which was subjected to a further examination. The physical examination at his diagnosis revealed giant splenomegaly (palpable 15 cm below costal margin). Blast count was $5.0 \%$ in peripheral blood and $7.3 \%$ in bone marrow. There was no additional chromosomal abnormality except for $\mathrm{t}(9 ; 22)(\mathrm{q} 34 ; \mathrm{q} 11.2)$ by chromosomal analysis. The patient's Sokal score was 1.5 indicating high risk, Hasford score was 1,332.4 indicating intermediate risk, EUTOS score was 134 indicating high risk, and ELTS score was 2.0877 indicating intermediate risk. A month after the diagnosis, the patient underwent TKI therapy comprising nilotinib up to $600 \mathrm{mg} /$ day, followed by $600 \mathrm{mg}$ bosu- 
Table 1. Laboratory data before tyrosine kinase inhibitor treatment

\begin{tabular}{|c|c|c|}
\hline WBC & 125,200 & $/ \mu \mathrm{L}$ \\
\hline Stab. & 11.5 & $\%$ \\
\hline Seg. & 31.0 & $\%$ \\
\hline Lym. & 3.5 & $\%$ \\
\hline Mono. & 0.0 & $\%$ \\
\hline Eos. & 6.0 & $\%$ \\
\hline Baso. & 6.5 & $\%$ \\
\hline Blast & 6.5 & $\%$ \\
\hline Promyelo. & 1.0 & $\%$ \\
\hline Myelo. & 26.5 & $\%$ \\
\hline Metamyelo. & 7.5 & $\%$ \\
\hline $\mathrm{RBC}$ & $327 \times 10^{4}$ & $/ \mu \mathrm{L}$ \\
\hline $\mathrm{Hb}$ & 9.3 & $\mathrm{~g} / \mathrm{dL}$ \\
\hline Hct & 30.4 & $\%$ \\
\hline MCV & 93.0 & $\mathrm{fL}$ \\
\hline MCHC & 30.6 & $\%$ \\
\hline PLT & $51.0 \times 10^{4}$ & $/ \mu \mathrm{L}$ \\
\hline CRP & 1.20 & $\mathrm{mg} / \mathrm{dL}$ \\
\hline TP & 6.0 & $\mathrm{~g} / \mathrm{dL}$ \\
\hline Alb & 4.1 & $\mathrm{~g} / \mathrm{dL}$ \\
\hline BUN & 15.2 & $\mathrm{mg} / \mathrm{dL}$ \\
\hline $\mathrm{Cr}$ & 0.68 & $\mathrm{mg} / \mathrm{dL}$ \\
\hline UA & 6.2 & $\mathrm{mg} / \mathrm{dL}$ \\
\hline T-Bil & 0.6 & $\mathrm{mg} / \mathrm{dL}$ \\
\hline GOT & 14 & $\mathrm{U} / \mathrm{L}$ \\
\hline GPT & 17 & $\mathrm{U} / \mathrm{L}$ \\
\hline ALP & 235 & $\mathrm{U} / \mathrm{L}$ \\
\hline$\gamma$-GTP & 28 & $\mathrm{U} / \mathrm{L}$ \\
\hline CPK & 12 & $\mathrm{U} / \mathrm{L}$ \\
\hline CHE & 181 & $\mathrm{U} / \mathrm{L}$ \\
\hline LDH & 601 & $\mathrm{U} / \mathrm{L}$ \\
\hline $\mathrm{Na}$ & 140 & $\mathrm{mmol} / \mathrm{L}$ \\
\hline $\mathrm{K}$ & 4.5 & $\mathrm{mmol} / \mathrm{L}$ \\
\hline $\mathrm{Cl}$ & 105 & $\mathrm{mmol} / \mathrm{L}$ \\
\hline PT & 70 & $\%$ \\
\hline PT-INR & 1.17 & \\
\hline APTT & 46.5 & $\mathrm{~s}$ \\
\hline FIB & 311 & $\mathrm{mg} / \mathrm{dL}$ \\
\hline ATIII & 72 & $\%$ \\
\hline FDP & 3 & $\mu \mathrm{g} / \mathrm{mL}$ \\
\hline D-dimer & 1.0 & $\mu \mathrm{g} / \mathrm{mL}$ \\
\hline
\end{tabular}

tinib, $45 \mathrm{mg}$ ponatinib, and $100 \mathrm{mg}$ dasatinib maximum daily dose. Laboratory data prior to TKI treatment are shown in Table 1. None of the TKIs exerted a clinical response, except for ponatinib, which yielded a hematological response (Fig. 1). No known mutations in the ABL1 kinase domain were detected after TKI therapy, prompting sequencing analysis. We performed targeted panel sequencing, by using prior-stem cell transplantation sample, which includes 377 genes implicated in myeloid malignancies. This analysis revealed a frameshift mutation in ASXL1 on chromosome 20q11. The patient underwent a stem cell transplantation with bone marrow donated by an unrelated HLA 7/8-matched (HLA-Cw1 locus mismatched, GVH direction) male from the Japan Marrow Donor Program. Tacrolimus and short-term methotrexate were used for graft-versus-host disease (GVHD) prophylaxis. On the 18th day after transplantation, the patient received neutrophil engraftment followed by reticulocyte engraftment 14 days later and platelet engraftment 22 days later. The patient achieved complete remission, with the bone marrow showing undetectable levels of $B C R-A B L 1$ mRNA 


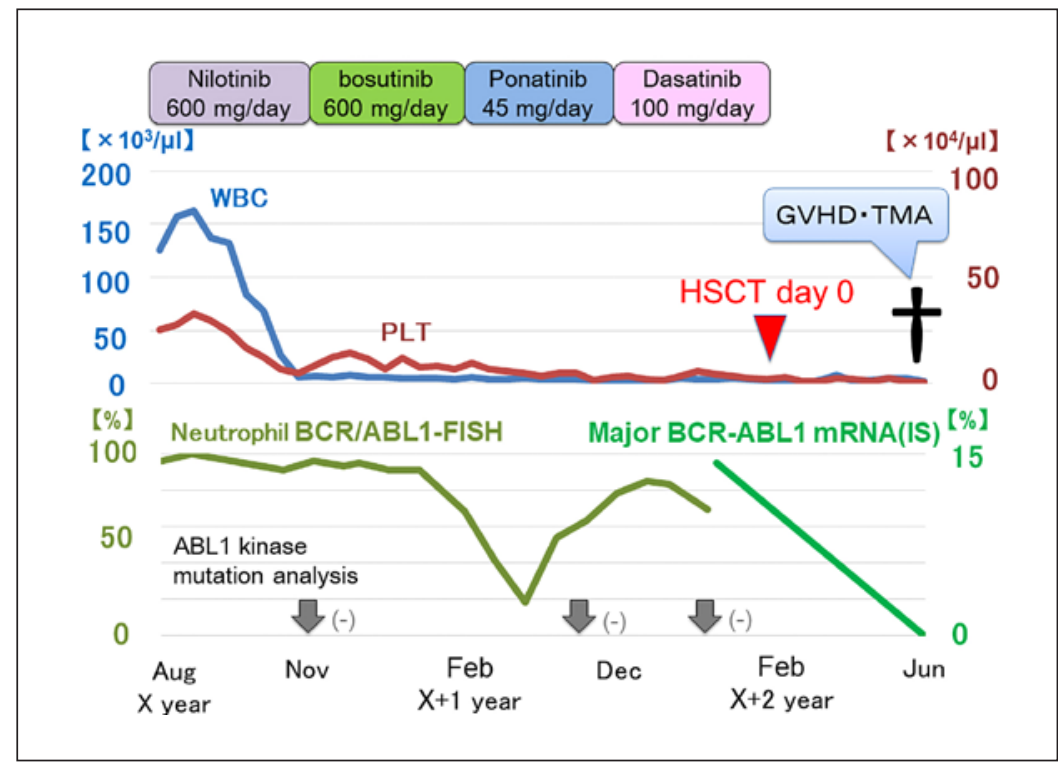

Fig. 1. The patient's clinical course. Nilotinib administration had an adverse effect on white blood cell and platelets, but did not diminish the $b c r-a b l$ fusion gene. The switch to bosutinib and dasatinib therapy did not change the response. The patient responded only to ponatinib treatment, which achieved a hematological response with $18 \%$ of $b c r-a b l 1$ FISH. However, no durable response was available. Hematopoietic stem cell transplantation yielded a molecular response without detection of major BCR-ABL1 mRNA by RT-PCR. The patient died of transplantation-related mortality, GVHD and TMA on the 121st day after transplantation. FISH, fluorescence in situ hybridization; GVHD, graft-versus-host disease; HSCT, hematopoietic stem cell transplantation; PLT, platelet; TMA, thrombotic microangiopathy; WBC, white blood cells.

by RT-PCR (International Scale). Despite treatment, the patient died on the 121st day from GVHD and thrombotic microangiopathy, which developed after the patient presented with GVHD.

\section{Discussion}

Physiologically, ASXL1 encodes a chromatin-binding protein involved in epigenetic regulation [12] by recruiting the polycomb repressive complex 2 (PRC2), a histone methyltransferase, which regulates gene activity by trimethylation of lysine 27 on histone 3 (H3K27me) $[13,14]$. Mutations in ASXL1 were originally reported and conferred poor prognosis in MDS [15] and chronic myelomonocytic leukemia (CMMoL) [5]. Frameshift mutations or nonsense mutations in exon 12 of ASXL1 abrogate protein expression [12] and consequently disrupt its function as a tumor suppressor, often in a variety of hematological malignancies [8]. Mutations in ASXL1 contribute to oncogenesis in hematopoietic cells, especially leukemogenesis, and promote myeloid transformation through loss of PRC2-mediated gene repression in MDS and CMMoL $[12,16]$.

ASXL1 mutations are common and associated with disease progression in acute myeloid leukemia (AML) [17] and similarly in CML, where ASXL1 mutations might be associated with poor prognosis and acute transformation [5]. Variation in prognosis and survival associated with ASXL1 mutations is seen across studies [18]. ASXL1 mutations are commonly associated with clonal hematopoiesis in healthy individuals [9-11], indicating that ASXL1 mutation may 
be a pre-leukemic event in hematopoiesis. Accumulating evidence points to a role for ASXL1 mutations in leukemogenesis during early hematopoietic events in many hematological malignancies, as described in AML [18] and CML [19,20]. In the latter case, mutations in ASXL1 occur early in CML stem cells, prior to bcr-abl translocation stage [19], as these cells clonally evolve [20]. This is considered an intrinsic event, rather than a bcr-abl fusion, which occurs after myeloid lineage differentiation [20].

In the two-hit model of AML development, class I mutation confers a proliferative or survival advantage, and class II mutations result in impaired myeloid differentiation as a secondary event [21]. Class II mutations in ASXL1 lead to its loss of function, impairing granulomonocytic differentiation in early human hematopoietic progenitors and contributing to leukemogenesis. Indeed, silencing of ASXL1 impairs the granulomonocytic lineage potential of human $\mathrm{CD}_{3} 4^{+}$progenitor cells by altering its gene expression profile, but not proliferation and apoptosis $[11,14]$. Alternatively, ASXL1 mutations might enhance other leukemogenesis pathways via other mechanisms, including epigenetic regulation.

ASXL1 plays a key role in epigenetic regulation of gene expression through methylation of histone H3K27, and disruption of ASXL1 drives myeloid malignancies [22]. Also, ASXL1 mutations may affect epigenetic regulation by inhibiting ubiquitination of lysine 119 at histone H2A (H2AK119). This may contribute to leukemogenesis though this remains to be proven [23]. Thus, even though a molecular mechanism for TKI resistance in ASXL1 mutation in CML has remained obscure, epigenetic modulation is a plausible mode of CML disease progression.

ASXL1 mutations occur early in CML stem cells, prior to $b c r-a b l$ translocation stage, and therefore, there might not be a high risk of treatment failure following TKI therapy [20]. The patient received hematopoietic stem cell transplantation and went into remission. This optimal response could have been prolonged without TKI maintenance therapy. Taken together, ASXL1 mutation might follow the HSCT trait as observed in this CML-CP case, which does not progress for years.

\section{Conclusions}

Whole exome sequence facilitated a clinical decision in this patient who went into remission. Though the clinical impact of $A S X L 1$ for CML is still under investigation, mutated ASXL1 possibly explain the TKI resistance mechanism. This case illustrated the necessity of HSCT in ASXL1 mutation-associated TKI-resistant CML-CP. In future, a discussion of CML with mutated $A S X L 1$ will facilitate treatment options.

\section{Statement of Ethics}

The Institutional Review Board approved the case report and submission of medical literature. We obtained written informed consent from the patient for participation in this study. We obtained consent to publish from the participants.

\section{Disclosure Statement}

The authors declare no competing interests. The authors declare no potential conflicts of interest. 
Imataki et al.: CML with ASXL1 Mutation

\section{Funding Sources}

Internal funding support is disclosed. This work was supported by internal funding, JSPS KAKENHI Grant Numbers JP16K19315, JP15K09570, and JP19K17927.

\section{Author Contributions}

O.I. and T.I. wrote the manuscript and made substantial contributions to concept and design; M.U., Y.N., K.K., and H.K. suggested important intellectual content and took part in the critical discussion; S.O. and N.K. managed the study and reviewed the manuscript; all authors read and approved the final version of the manuscript.

\section{Availability of Data and Material}

There are no other data analyzed in this study.

\section{References}

1 Cortes J, Rea D, Lipton JH. Treatment-free remission with first- and second-generation tyrosine kinase inhibitors. Am J Hematol. 2019 Mar;94(3):346-57.

2 Ren R. Mechanisms of BCR-ABL in the pathogenesis of chronic myelogenous leukaemia. Nat Rev Cancer. 2005 Mar;5(3):172-83.

3 Patel AB, O'Hare T, Deininger MW. Mechanisms of resistance to ABL kinase inhibition in chronic myeloid leukemia and the development of next generation ABL Kinase inhibitors. Hematol Oncol Clin North Am. 2017 Aug;31(4):589-612.

4 Abdel-Wahab 0, Figueroa ME. Interpreting new molecular genetics in myelodysplastic syndromes. Hematol Am Soc Hematol Educ Program. 2012;2012:56-64.

5 Gelsi-Boyer V, Trouplin V, Adélaïde J, Bonansea J, Cervera N, Carbuccia N, et al. Mutations of polycomb-associated gene ASXL1 in myelodysplastic syndromes and chronic myelomonocytic leukaemia. Br J Haematol. 2009 Jun;145(6):788-800.

6 Tefferi A, Pardanani A. Myeloproliferative neoplasms: a contemporary review. JAMA Oncol. 2015 Apr;1(1): 97-105.

7 Grossmann V, Kohlmann A, Zenger M, Schindela S, Eder C, Weissmann S, et al. A deep-sequencing study of chronic myeloid leukemia patients in blast crisis (BC-CML) detects mutations in $76.9 \%$ of cases. Leukemia. 2011 Mar; 25(3):557-60.

8 Togasaki E, Takeda J, Yoshida K, Shiozawa Y, Takeuchi M, Oshima M, et al. Frequent somatic mutations in epigenetic regulators in newly diagnosed chronic myeloid leukemia. Blood Cancer J. 2017 Apr 28;7(4):e559.

9 Genovese G, Kähler AK, Handsaker RE, Lindberg J, Rose SA, Bakhoum SF, et al. Clonal hematopoiesis and bloodcancer risk inferred from blood DNA sequence. N Engl J Med. 2014 Dec 25;371(26):2477-87.

10 Jaiswal S, Fontanillas P, Flannick J, Manning A, Grauman PV, Mar BG, et al. Age-related clonal hematopoiesis associated with adverse outcomes. N Engl J Med. 2014 Dec 25;371(26):2488-98.

11 Xie M, Lu C, Wang J, McLellan MD, Johnson KJ, Wendl MC, et al. Age-related mutations associated with clonal hematopoietic expansion and malignancies. Nat Med. 2014 Dec;20(12):1472-8.

12 Pellagatti A, Boultwood J. The molecular pathogenesis of the myelodysplastic syndromes. Eur J Haematol. 2015 Jul;95(1):3-15.

13 Abdel-Wahab O, Adli M, LaFave LM, Gao J, Hricik T, Shih AH, et al. ASXL1 mutations promote myeloid transformation through loss of PRC2-mediated gene repression. Cancer Cell. 2012 Aug 14;22(2):180-93.

14 Davies C, Yip BH, Fernandez-Mercado M, Woll PS, Agirre X, Prosper F, et al. Silencing of ASXL1 impairs the granulomonocytic lineage potential of human CD34+ progenitor cells. Br J Haematol. 2013 Mar;160(6):84250.

15 Bejar R, Stevenson K, Abdel-Wahab O, Galili N, Nilsson B, Garcia-Manero G, et al. Clinical effect of point mutations in myelodysplastic syndromes. N Engl J Med. 2011 Jun 30;364(26):2496-506.

16 Gelsi-Boyer V, Brecqueville M, Devillier R, Murati A, Mozziconacci MJ, Birnbaum D. Mutations in ASXL1 are associated with poor prognosis across the spectrum of malignant myeloid diseases. J Hematol Oncol. $2012 \mathrm{Mar}$ $21 ; 5: 12$. 
17 Boultwood J, Perry J, Pellagatti A, et al. Frequent mutation of the polycomb-associated gene ASXL1 in the myelodysplastic syndromes and in acute myeloid leukemia. Leukemia. 2010;24:1062-5.

18 Eriksson A, Lennartsson A, Lehmann S. Epigenetic aberrations in acute myeloid leukemia: early key events during leukemogenesis. Exp Hematol. 2015 Aug;43(8):609-24.

19 Boultwood J, Perry J, Zaman R, Fernandez-Santamaria C, Littlewood T, Kusec R, et al. High-density single nucleotide polymorphism array analysis and ASXL1 gene mutation screening in chronic myeloid leukemia during disease progression. Leukemia. 2010 Jun;24(6):1139-45.

20 Kim T, Tyndel MS, Kim HJ, Ahn JS, Choi SH, Park HJ, et al. Spectrum of somatic mutation dynamics in chronic myeloid leukemia following tyrosine kinase inhibitor therapy. Blood. 2017 Jan 5;129(1):38-47.

21 Gilliland DG. Molecular genetics of human leukemias: new insights into therapy. Semin Hematol. 2002 Oct; 39(4 Suppl 3):6-11.

22 Inoue D, Fujino T, Sheridan P, Zhang YZ, Nagase R, Horikawa S, et al. A novel ASXL1-OGT axis plays roles in H3K4 methylation and tumor suppression in myeloid malignancies. Leukemia. 2018 Jun;32(6):1327-37.

23 Inoue D, Fujino T, Kitamura T. ASXL1 as a critical regulator of epigenetic marks and therapeutic potential of mutated cells. Oncotarget. 2018 Oct 16;9(81):35203-4. 